\title{
Square Planar 4 Molecular Geometry
}

National Cancer Institute

\section{Source}

National Cancer Institute. Square Planar 4 Molecular Geometry. NCI Thesaurus. Code C103214.

Square planar molecular geometry where the Cahn-Ingold-Prelog (CIP) priority of the donor atom directly across from the priority 1 donor atom is 4 . 